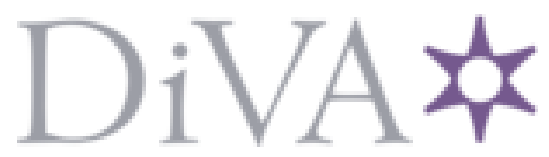

http://www.diva-portal.org

This is the published version of a paper published in International psychogeriatrics.

Citation for the original published paper (version of record):

Lövheim, H., Sandman, P., Kallin, K., Karlsson, S., Gustafson, Y. (2006)

Relationship between antipsychotic drug use and behavioral and psychological symptoms of dementia in old people with cognitive impairment living in geriatric care..

International psychogeriatrics, 18(4): 713-726

http://dx.doi.org/10.1017/S1041610206003930

Access to the published version may require subscription.

N.B. When citing this work, cite the original published paper.

Permanent link to this version:

http://urn.kb.se/resolve?urn=urn:nbn:se:umu:diva-22324 


\title{
Relationship between antipsychotic drug use and behavioral and psychological symptoms of dementia in old people with cognitive impairment living in geriatric care
}

\author{
Hugo Lövheim, ${ }^{1}$ Per-Olof Sandman, ${ }^{2}$ Kristina Kallin, \\ Stig Karlsson ${ }^{1}$ and Yngve Gustafson ${ }^{1}$ \\ ${ }^{1}$ Department of Community Medicine and Rehabilitation, Geriatric Medicine, Umeå University, Umeå, Sweden \\ ${ }^{2}$ Department of Nursing, Umeå University, Umeå, Sweden
}

ABSTRACT

Background: Behavioral and psychological symptoms of dementia (BPSD) commonly occur among cognitively impaired people in geriatric care. BPSD are often managed with antipsychotic drugs, despite the associated serious health risks. The aim of the present study was to discover factors associated with the use of antipsychotics.

Methods: A cross-sectional study in all geriatric care units in the county of Västerbotten, Sweden, which included 2017 residents aged 65 years and over with cognitive impairment (mean age was 83.5 years). Data were collected from prescription records and observations made by care staff of BPSD among residents during the preceding week. A multivariate regression model was constructed to find factors independently associated with antipsychotic drug use.

Results: Eleven factors were independently associated with the use of antipsychotics. Aggressive, verbally disruptive and wandering behavior, hallucinatory and depressive symptoms, male sex, living in a group dwelling for people with dementia, imposed mental workload, the ability to rise from a chair, activities of daily living (ADL) dependency and lower age all correlated significantly.

Conclusions: Antipsychotic drug treatment of old people with cognitive impairment in geriatric care is common, and determined not only by the patient's symptoms but also by factors related more closely to the caregiver and the caring situation. These findings raise important questions about the indications for drug treatment in relation to the patient's quality of life. 
Key words: residential care, nursing home, dementia, BPSD, aggressiveness, sex difference, pharmacological treatment

\section{Introduction}

A person with a dementia disorder often exhibits behavioral and psychological symptoms in addition to cognitive decline (Margallo-Lana et al., 2001). The term "behavioral and psychological symptoms of dementia" (BPSD) is not a diagnostic entity but has been used to describe the broad spectrum of behavioral changes (e.g. aggressiveness, agitation and wandering behavior) and psychological symptoms (e.g. mood changes, suspiciousness and hallucinations) that can be seen in someone affected by dementia.

These behaviors and psychological symptoms are very common in populations of old people with dementia and the prevalence of BPSD in different studies varies between $79 \%$ and approximately 92\% (Brodaty et al., 2001; Margallo-Lana et al., 2001; Pitkala et al., 2004). Some of the symptoms increase the difficulty of caring for the person with dementia, and there is a correlation between BPSD and burden of care, and even with caregiver depression (Black and Almeida, 2004).

It is not uncommon in clinical practice for people with BPSD to be treated pharmacologically, often with antipsychotics (Margallo-Lana et al., 2001; Pitkala et al., 2004). The introduction of atypical antipsychotics, such as risperidone and olanzapine, has changed the treatment for this population, as they are more effective in controlling BPSD and have fewer and milder extrapyramidal side-effects (EPS) than conventional antipsychotics (Brodaty et al., 2003; De Deyn et al., 1999). In the USA the atypical antipsychotics have now become a more common treatment for old people with dementia than the conventional drugs (Liperoti et al., 2003). However, even if the atypicals seem to have a more advantageous side-effect profile than the conventional, they are not free of side-effects. In the treatment of frail old people, EPS, sedation and cognitive decline are seen even when atypical antipsychotics are used. It has also been reported that their use incurs an approximately threefold increase in the risk of adverse cerebrovascular events (stroke or transitory ischemic attack) compared to when a placebo drug is administered (Brodaty et al., 2003). A mortality rate of $3.5 \%$, compared to $1.5 \%$ for the placebo group, has been seen in clinical trials (Katz, 2004). Concerns have been raised about whether treating BPSD with antipsychotics really improves the quality of life of the patient (Ballard and Margallo-Lana, 2004; Brylewski and Duggan, 1999).

Studies have described and determined some factors that correlate with the prescription of antipsychotics to old people with dementia living in institutional 
care. Psychotic features, agitation, offensive behavior, nocturnal disturbances, better mobility, lower age and higher body mass index have all been found to correlate with an increased risk of being treated with antipsychotics in various studies. Cognitive function and activities of daily living (ADL) dependency correlated negatively in some and positively in others (Craig et al., 2003; Lindesay et al., 2003; Robertson et al., 2000; Sørensen et al., 2001). The complex process that leads to an old person with dementia being given antipsychotic treatment, however, remains far from being fully understood.

The aims of the present study were: (a) to describe the characteristics of a group of old people with cognitive impairment in geriatric care who were treated with antipsychotics, and (b) to discover whether there were any associations between the use of antipsychotics and BPSD, and other factors linked both to the person and to the geriatric care environment.

\section{Methods}

\section{Materials}

The present study is a cross-sectional questionnaire survey performed in May 2000 in the county of Västerbotten in northern Sweden. At that time the population of the area numbered approximately 256000 people, of whom 44211 $(17.3 \%)$ were aged 65 years or older.

A form was sent to all the geriatric care settings in the county (68 residential care facilities, 31 nursing homes, 66 group dwellings for people with dementia, seven rehabilitation/short-stay units, two somatic geriatric and two psychogeriatric clinics), which the staff were asked to complete and return to the researchers. The staff received written instructions about how to complete the assessments and were informed that members of the research team could be contacted by phone to answer questions or provide additional guidance. The particular member of staff who knew the resident best was asked to fill in the assessment scales. The staff were informed that their assessments should be based on observations of the resident's state during the preceding week (i.e. the observation period should be 1 week). Members of the research group coded the answers into a data file.

The investigated population comprised a total of 4357 people. The forms were completed and returned for 3804 (87.3\%) individuals. Six units did not participate for various reasons including understaffing and some single cases were also not assessed due, for example, to too short an observation period. Subsequently, 466 cases were excluded because they were younger than 65 years or because incomplete data were returned concerning age, sex or cognitive 
impairment. From the remaining population of 3338 cases, we selected those with cognitive impairment, a total of 2017 persons. These people became the study population.

The study was approved by the Ethics Committee of the Faculty of Medicine at Umeå University (registration number 00-170).

\section{Procedures}

The Multi-Dimensional Dementia Assessment Scale (MDDAS) (Sandman et al., 1988) was used to make the assessments. This scale measures, for example, mobility, function in ADL, orientation and BPSD, and also includes a registration of current drug prescriptions. The MDDAS has good intra- and inter-rater reliability (Sandman et al., 1988).

A registered nurse filled in the data concerning the prescribed medication, taking it from the resident's prescription record. In the coding of the drug prescriptions, carried out by members of the research team, the drugs were subsumed into a number of standard groups (e.g. anxiolytics, analgesics and antipsychotics) and each person in the study population was assigned a dichotomous value for each standard group or single substance. This meant that no information about doses was coded. Pro re nata ("as needed") medication was not coded either, hence only figures for ongoing medication are given. Antipsychotic drugs (ATC code N05A) (World Health Organization, 2005) were further divided into conventional (e.g. haloperidol) and atypical (e.g. risperidone and olanzapine).

An ADL score was calculated based on the resident's ability to cope with dressing, hygiene, eating, bladder and bowel control. All ADL categories score 1-5, except the bladder control category, which scores $0-4$. Hence the ADL score can vary between 4 and 24, where a higher score indicates greater ADL independence. The physical and mental workloads of the caregiver generated by the resident were assessed on a five-point scale from minimum to maximum workload, where a higher score means a greater workload.

Cognitive impairment was measured using a subscale developed by Gottfries and Gottfries (published in Adolfsson et al., 1981). The scale consists of 27 items that measure the person's ability to orient her/himself. Scores of less than 24 are considered to indicate cognitive impairment, which correlates with a sensitivity of $90 \%$ and a specificity of $91 \%$ (Adolfsson et al., 1981) to the cut-off of 24/30 traditionally used in the Mini-mental State Examination (Folstein et al., 1975). The residents who scored 24 or less were designated cognitively impaired even though the majority of them probably had a dementia disorder. The dichotomous variable cognitive impairment was used to select cases for the analysis, but the raw orientation score was used in the analysis itself. 
The MDDAS contains 25 behavioral items and 14 different psychological symptoms, which have been described in detail elsewhere (Adolfsson et al., 1981). Each item is rated on a three-point scale to indicate whether the symptom or behavior occurs at least once a day, once a week or never. For each item the person was assigned the value 3, 1 or 0 , respectively. It has previously been shown that these BPSD items can be grouped together to represent clusters of symptoms/behaviors, which can then be labeled and used for further analysis (Näsman et al., 1993; Kallin et al., 2005). The same labels are used in the present study.

\section{Statistics}

Dichotomous variables were analyzed using Pearson's $\chi^{2}$, continuous variables with the independent variable $t$-test for variables that met the assumption of normal distribution, and the non-parametric Mann-Whitney $U$-test for the others. Only the distribution of the continuous variables age and total number of drugs were approximately normal, as determined by visual examination.

Both the behavioral items and the psychological symptom items of the MDDAS were reduced to fewer factors by means of a Varimax principal component analysis. We chose to extract factors with eigenvalues of above 1.00, producing factors that conveyed meaningful information. The rotated solution factors were saved as a $z$-score, that is a standardized score with a population mean of zero and a standard deviation of one. This also means that all items contributed to all factors, but with different loadings, or weights. Factor loading, which ranges from -1 to 1 , is equal to the item's correlation to the factor. The result is presented in Table 3. The first column shows how many of the patients were assessed as having displayed the behavior or symptom at least once a week. Under each factor only the items with a loading above 0.5 are presented. The models explained $50.9 \%$ of the total variance of the behavioral symptoms and $66.8 \%$ of the psychological symptom variance. The groups of variables were tested for internal consistency using a Cronbach $\alpha$ analysis. $\alpha$ values ranged from 0.40 to 0.73 . Finally, a multivariate logistic regression model was constructed to reveal the variables and factors that were independently associated with the use of antipsychotic drugs. The behavioral symptom factors and the psychological symptom factors from the principal component analysis, the data on accommodation type, ADL dependency, orientation score, age, sex, mental workload and whether or not the person could rise from a chair unaided were entered into the regression. Because of a high bivariate correlation between physical workload and ADL score, the former was omitted from the analysis. For the same reason the variable "ability to walk independently" was also omitted.

All data handling and statistical calculations were performed with the Statistical Package for Social Science version 11.0 (for MAC OSX). 


\section{Results}

Table 1 presents a comparison between people who were prescribed an antipsychotic drug and those who were not. Those who took antipsychotic drugs were more often men, younger, more ADL dependent and had on average a lower orientation score. They were also more mobile and imposed a greater mental workload on the staff. They more often lived in a group dwelling for people with dementia and less often in residential care.

The use of different classes of psychotropic drugs is presented in Table 2. Atypical antipsychotics accounted for about half of the antipsychotics prescribing.

There were six behavioral symptom extracted factors and these were labeled "aggressive behavior," "restless behavior," "wandering behavior," "escape behavior," "verbally disruptive/attention-seeking behavior" and "regressive/ inappropriate behavior." There were five psychological symptom extracted factors, which were labeled "depressive symptoms," "paranoid symptoms," "hallucinatory symptoms," "hyperactive symptoms" and "passiveness." The result of the factor analysis is shown in Table 3.

The multivariate regression model is shown in Table 4. Eleven different factors correlated significantly with the use of antipsychotics. Male sex, living in a group dwelling for people with dementia, and the ability to rise from a chair unaided were associated significantly with the use of antipsychotics. The odds ratio assigned to the behavior and psychological symptom factors is to be interpreted as meaning that one standard deviation change in, for example, aggressiveness increase the odds of being prescribed antipsychotics by $33 \%$. Three different behavior factors and two psychological symptom factors correlated significantly. Mental workload, ADL independence and age were also associated significantly with the prescribing of antipsychotics. An individual's orientation score did not correlate with the use of antipsychotics, neither as a continuous variable nor when divided into larger groups (data not shown). The model produced a concordance between observed and predicted values of $76.0 \%$, and a Cox and Snell $R^{2}$ of 0.12 .

\section{Discussion}

The prevalence of antipsychotic medication in this study is in line with, or somewhat lower than, that reported previously (Craig et al., 2003; Pitkala et al., 2004; Sørensen et al., 2001). Different population characteristics and methodology make closer comparisons impossible. An equal number of patients used atypical and conventional antipsychotics and the proportion of the former will probably rise even more in the future. 
Table 1. Comparison between patients with and without antipsychotic drug treatment

\begin{tabular}{|c|c|c|c|c|}
\hline & $\begin{array}{l}\text { ANTIPSYCHOTICS } \\
\text { NON-USER }\end{array}$ & $\begin{array}{l}\text { ANTIPSYCHOTICS } \\
\text { USER* }\end{array}$ & $p$-VALUE & TOTAL \\
\hline Cases, $n(\%)$ & $1486(73.7)$ & $531(26.3)$ & & $2017(100.0)$ \\
\hline Women, $n(\%)$ & $1060(71.3)$ & $334(62.9)$ & $<0.001$ & $1394(69.1)$ \\
\hline Age, years, mean $\pm S D$ & $83.7 \pm 6.68$ & $82.7 \pm 7.08$ & 0.004 & $83.5 \pm 6.80$ \\
\hline ADL score $(4-24)$, mean $\pm S D$ & $13.1 \pm 6.21$ & $12.4 \pm 5.66$ & 0.065 & $12.9 \pm 6.07$ \\
\hline Orientation score $(0-27)$, mean \pm SD & $11.4 \pm 7.13$ & $10.7 \pm 6.48$ & 0.030 & $11.2 \pm 6.97$ \\
\hline Walks independently, $n(\%)$ & $558(38.2)$ & $227(43.6)$ & 0.030 & $785(39.6)$ \\
\hline Can rise from chair unaided, $n(\%)$ & $794(54.2)$ & $328(62.4)$ & 0.001 & $1122(56.4)$ \\
\hline Confined to bed, $n(\%)$ & $67(4.6)$ & $13(2.5)$ & 0.037 & $80(4.1)$ \\
\hline Impaired vision, $n(\%)$ & $212(14.7)$ & $93(17.9)$ & 0.085 & $305(15.6)$ \\
\hline Impaired hearing, $n(\%)$ & $157(10.9)$ & $60(11.5)$ & 0.688 & $217(11.0)$ \\
\hline Physical workload (1-5), mean \pm SD & $3.0 \pm 1.44$ & $3.0 \pm 1.38$ & 0.970 & $3.0 \pm 1.42$ \\
\hline Mental workload $(1-5)$, mean \pm SD & $2.5 \pm 1.28$ & $3.2 \pm 1.26$ & $<0.001$ & $2.7 \pm 1.31$ \\
\hline Group dwelling, $n(\%)$ & $392(26.4)$ & $213(40.1)$ & $<0.001$ & $605(30.0)$ \\
\hline Nursing home, $n(\%)$ & $452(30.4)$ & $159(29.9)$ & 0.838 & $611(30.3)$ \\
\hline Rehabilitation/short-stay unit, $n(\%)$ & $23(1.5)$ & $6(1.1)$ & 0.488 & $29(1.4)$ \\
\hline Residential care, $n(\%)$ & $572(38.5)$ & $141(26.6)$ & $<0.001$ & $713(35.3)$ \\
\hline Somatic geriatric clinic, $n(\%)$ & $19(1.3)$ & $4(0.8)$ & 0.328 & $23(1.1)$ \\
\hline Psychogeriatric clinic, $n(\%)$ & $28(1.9)$ & $8(1.5)$ & 0.573 & $36(1.8)$ \\
\hline
\end{tabular}

$\mathrm{SD}=$ standard deviation; $\mathrm{ADL}=$ activities of daily living.

*Patients with ongoing medication with at least one antipsychotic drug (ATC code N05A). 
Table 2. Prevalence of psychotropic drug use

\begin{tabular}{ll}
\hline & $n(\%)$ \\
\hline Any antipsychotics (N05A) & $531(26.3)$ \\
Conventional antipsychotics & $285(14.1)$ \\
Atypical antipsychotics & $270(13.4)$ \\
Anti-anxiety drugs (N05B) & $273(13.5)$ \\
Hypnotics and sedatives (N05C) & $640(31.7)$ \\
Antidepressants (N06A) & $873(43.3)$ \\
\hline
\end{tabular}

Table 3. Factor analysis of behavioral and psychological symptoms

$\begin{array}{lll} & & \text { VARIANCE } \\ (\%) & \text { FACTOR } & \text { EXPLAINED } \\ \text { LOADING } & (\%)\end{array}$

Behavior items

Aggressive behavior

Hits patients/staff

Aggressive threats (words or gestures)

to patients/staff

Resists being dressed and undressed

Spits out drugs

Shrieks and shouts continuously

Wandering behavior

Lies in other patients' beds

Piles up chairs, pushes tables, upends furniture

Takes things from other patients' boxes and closets

Restless behavior

Mixes up food

Rolls up tablecloths

Eats soil from potted plants, cigarette ends, etc.

Tears up newspaper, etc.

Eats others' food

$\begin{array}{ll}16.9 & 0.82\end{array}$

10.8

$36.3 \quad 0.78$

$36.9 \quad 0.69$

$22.7 \quad 0.57$

$21.9 \quad 0.51$

Escape behavior

Packs up his/her things, is often on the way home

Hides things

Often stands at the outer door wanting to go out

Verbally disruptive/attention seeking

Constantly seeks attention of the staff

Talks spontaneously with patients/staff Interrupted night's sleep

$\begin{array}{ll}5.6 & 0.63 \\ 8.4 & 0.58\end{array}$

$8.4 \quad 0.58$

$10.5 \quad 0.57$

$\begin{array}{ll}21.9 & 0.69\end{array}$

$21.1 \quad 0.62$

$2.9 \quad 0.60$

$10.4 \quad 0.59$

$8.7 \quad 0.58$

$13.4 \quad 0.71$

$23.7 \quad 0.70$

$15.7 \quad 0.56$

$54.5 \quad 0.76$

$\begin{array}{ll}72.4 & 0.67\end{array}$

$56.7 \quad 0.54$
9.9 
Table 3. Continued.

\begin{tabular}{|c|c|c|c|}
\hline & $(\%)$ & $\begin{array}{l}\text { FACTOR } \\
\text { LOADING }\end{array}$ & $\begin{array}{l}\text { VAR I A N C E } \\
\text { EX P L A I N E D } \\
(\%)\end{array}$ \\
\hline Regressive/inappropriate behavior & & & 5.0 \\
\hline Smears feces on clothes, furniture, etc. & 13.0 & 0.82 & \\
\hline $\begin{array}{l}\text { Urinates in wastepaper baskets, wash-basins } \\
\text { or on the floor }\end{array}$ & 9.5 & 0.52 & \\
\hline Cumulative variance explained & & & 50.9 \\
\hline \multicolumn{4}{|c|}{ Items that did not contribute more than 0.5 to any of the factors } \\
\hline Undresses in the dayroom & 7.6 & & \\
\hline Unruly in bed, throws bedclothes on floor & 20.6 & & \\
\hline $\begin{array}{l}\text { Wanders back and forth alone or with other } \\
\text { patients }\end{array}$ & 29.2 & & \\
\hline Does not want to go to bed & 21.9 & & \\
\hline \multicolumn{4}{|l|}{ Psychological symptom items } \\
\hline Depressive symptoms & & & 16.1 \\
\hline Sad & 53.2 & 0.85 & \\
\hline Crying & 27.0 & 0.83 & \\
\hline Fearful & 43.4 & 0.59 & \\
\hline Hallucinatory symptoms & & & 13.8 \\
\hline Hallucinates auditorially & 16.3 & 0.90 & \\
\hline Hallucinates visually & 23.8 & 0.87 & \\
\hline Speaks to her/himself & 41.6 & 0.53 & \\
\hline Paranoid symptoms & & & 12.9 \\
\hline Easily annoyed & 42.3 & 0.84 & \\
\hline Suspicious & 34.9 & 0.82 & \\
\hline Passivity & & & 12.4 \\
\hline Does not cooperate & 54.7 & 0.85 & \\
\hline Lacks initiative & 79.8 & 0.82 & \\
\hline Does not seek help & 45.9 & 0.50 & \\
\hline Hyperactive symptoms & & & 11.6 \\
\hline Overactive/manic & 15.2 & 0.83 & \\
\hline Disturbed and restless & 57.2 & 0.68 & \\
\hline Cumulative variance explained & & & 66.8 \\
\hline \multicolumn{4}{|c|}{ Items that did not contribute more than 0.5 to any of the factors } \\
\hline Complains & 41.9 & & \\
\hline
\end{tabular}

The logistic regression revealed 11 factors that were independently associated with the use of antipsychotic drugs. That male sex was associated with antipsychotic treatment cannot be explained by the fact that men might be more aggressive and more agitated, because behavioral and psychological symptoms are also included in the analysis, and are therefore compensated for. Thus we must conclude that there is some factor, which is neither behavior nor psychological morbidity, that makes physicians more likely to prescribe 
Table 4. Multivariate logistic regression of antipsychotic drug use

\begin{tabular}{|c|c|c|c|}
\hline & $\begin{array}{l}\text { ODDS } \\
\text { RATIO }\end{array}$ & $\begin{array}{l}95 \% \text { CONFIDENCE } \\
\text { INTERVAL }\end{array}$ & $p-\mathrm{VALUE}$ \\
\hline ADL score & 0.97 & $0.94-0.99$ & 0.007 \\
\hline Age & 0.98 & $0.96-0.99$ & 0.043 \\
\hline Aggressive behavior & 1.31 & $1.15-1.50$ & $<0.001$ \\
\hline Can rise from chair unaided & 1.68 & $1.19-2.36$ & 0.003 \\
\hline Depressive symptoms & 1.15 & $1.02-1.30$ & 0.019 \\
\hline Group dwelling & 1.39 & $1.07-1.81$ & 0.006 \\
\hline Hallucinatory symptoms & 1.21 & $1.08-1.35$ & 0.001 \\
\hline Male sex & 1.40 & $1.08-1.82$ & 0.013 \\
\hline Mental workload & 1.26 & $1.13-1.40$ & $<0.001$ \\
\hline Verbally disruptive behavior & 1.25 & $1.09-1.43$ & 0.002 \\
\hline Wandering behavior & 1.19 & $1.06-1.34$ & 0.003 \\
\hline
\end{tabular}

$\mathrm{ADL}=$ activities of daily living; higher score $=$ more independent.

Model Cox and Snell $R^{2}=0.12$, concordance between observed and predicted value $=0.76$.

antipsychotics to men. Tradition and prejudice might play a role here. Another explanation may be that even if men and women are equally aggressive, the staff in the geriatric care setting may think the former constitute a greater problem as they may perhaps be stronger and therefore more threatening. That the ability to rise from a chair unaided was correlated to the use of antipsychotics also indicates that antipsychotic medication is used if the staff feel threatened or have problems controlling the old person with dementia. The factor "living in a group dwelling for people with dementia" was also an associated factor. The group dwellings, small units with relatively high staffing rates, provide specialized care for people with a dementia illness. It is therefore somewhat surprising that a more frequent use of antipsychotic drugs occurs in this type of housing, although such a setting offers the possibilities for managing behavioral problems non-pharmacologically. A tradition of managing behavior pharmacologically may be an explanation.

It is not surprising that imposed mental workload correlated with antipsychotic medication, as such a correlation has previously been shown between staff workload and behavioral problems (Black and Almeida, 2004). All these four associated factors, however, raise the question of whether the patients are treated for their own sake, or for that of the staff. In light of the known side-effects and rather serious risks associated with antipsychotic drug use (Brodaty et al., 2003; Katz, 2004), this is an important ethical question, especially because there are alternative ways of managing the behavioral disturbances that might occur in dementia. Non-pharmacological approaches have been used successfully to reduce behavioral disturbances (Rovner et al., 1996; Sloane et al., 2004). Furthermore, most studies proving the effectiveness of antipsychotics have been 
performed over shorter periods of time, while studies lasting over a whole year (Ballard et al., 2001) and discontinuation studies (Ballard et al., 2004; Thapa et al., 1994) have found no benefits from ongoing antipsychotic medication for a majority of patients.

Aggressive behavior, verbally disruptive/attention-seeking behavior and wandering behavior were the types of behavior that correlated with antipsychotic use. This might have been expected, as the efficacy of antipsychotic drug treatment, compared to a placebo, for these behavioral disturbances has been proved (Brodaty et al., 2003; De Deyn et al., 1999). The effectiveness of antipsychotic drugs in dementia is, however, modest (Gormley and Howard, 1999). Concerning the psychological symptoms, the correlation with hallucinatory symptoms is uncontroversial, as hallucinations are a wellestablished indication for antipsychotic treatment. The correlation with depression, however, might indicate an adverse side-effect rather than serve as an indication for treatment. Finally, the use of antipsychotic drugs was correlated with lower age and higher ADL dependency. For age and ADL dependency, it should be borne in mind that the odds ratio is for a 1-year change or one point on the ADL scale. For example, a change in age of 10 years gives an odds ratio of $0.98^{10}=0.82$.

The orientation score did not have a significant influence on the prescription of antipsychotic drugs. The conclusion is that it is factors other than the person's cognitive level that lead to treatment with antipsychotics.

When used as a clinical outcome measure, the term BPSD may lead to an indication for antipsychotic drug use that is too broad. Even if it is shown that antipsychotic drugs produce amelioration in some measurements of behavior and psychological symptoms, it does not follow that antipsychotics are good for all kinds of symptoms. The etiology of behavioral and psychological symptoms in dementia is not the same as in schizophrenia, and a serotonin deficit is thought to be important (Herrmann and Lanctôt, 1997). Treatment with serotonin receptor antagonists (like the atypical antipsychotics) seems irrational from that point of view, and serotonin-specific reuptake inhibitors (SSRIs) have also been shown to have a better effect on aggressive behavior than antipsychotic agents (Pollock et al., 2002). There is still a need for randomized studies comparing the efficacy of different classes of psychotropics in the treatment of behavioral disturbances (e.g. atypical neuroleptics compared to SSRIs) and the determination of subgroups of behavior that respond to specific treatments.

The factor analysis was used to reduce the number of factors to be entered into the multivariate regression. Such a reduction always entails a significant loss of information, as indicated by the model's degree of variance explanation, which was $50.9 \%$ for the behavioral factors and $66.8 \%$ for the psychological symptoms. The loss of information is a consequence of reducing the number of factors. 
The rationale for this is that too many intercorrelated factors in a multivariate regression weakens it, or even makes it impossible to determine the contributing factors. As the factors in a rotated factor analysis solution are, by definition, uncorrelated, they are well suited to a multivariate regression. In addition, the grouping of the items into factors contains in itself useful information, as it shows which behaviors correlate with each other and may be regarded as a syndrome.

The regression model has a rather low explanatory value for the use of antipsychotics, indicating that a large number of random and/or hardly controlled factors also make a contribution. This does not interfere with the interpretation of the findings. People with missing values for behavioral and psychological items were excluded from the factor analysis. We estimate that these missing values were randomly distributed and do not affect the results.

\section{Conclusions}

Antipsychotic drug treatment of old people with cognitive impairment in geriatric care is common and is determined not only by the patient's symptoms but also by factors related more closely to the caregiver and the caring situation. This finding raises important questions about the indications for drug treatment in relation to the patient's quality of life. Both further improvement of non-pharmacological management and increased knowledge about different pharmacological strategies are needed.

\section{Conflict of interest}

None.

\section{Description of authors' roles}

H. Lövheim and Y. Gustafson collected and analyzed the data and wrote the paper. P. O. Sandman and S. Karlsson designed the study, recruited the subjects and collected, analyzed and interpreted the data. K. Kallin reviewed the data and wrote the paper.

\section{Acknowledgments}

This study was supported by grants from the Umeå University Foundation for Medical Research, the Swedish Foundation for Healthcare Sciences and Allergy Research, the Field Research Center for the Elderly in Västerbotten and Erik and Anne-Marie Detlof's Foundation, Umeå University. 


\section{References}

Adolfsson, R., Gottfries, C. G., Nyström, L. and Winblad, B. (1981). Prevalence of dementia disorders in institutionalized Swedish old people. The work load imposed by caring for these patients. Acta Psychiatrica Scandinavica, 63, 225-244.

Ballard, C. and Margallo-Lana, M. (2004). The relationship between antipsychotic treatment and quality of life for patients with dementia living in residential and nursing home care facilities. Fournal of Clinical Psychiatry, 65(Suppl. 11), 23-28.

Ballard, C. et al. (2001). A 1-year follow-up study of behavioral and psychological symptoms in dementia among people in care environments. Fournal of Clinical Psychiatry, 62, 631-636.

Ballard, C. et al. (2004). A 3-month, randomized, placebo-controlled, neuroleptic discontinuation study in 100 people with dementia: the neuropsychiatric inventory median cutoff is a predictor of clinical outcome. Fournal of Clinical Psychiatry, 65, 114-119.

Black, W. and Almeida, O. (2004). A systematic review of the association between the behavioral and psychological symptoms of dementia and burden of care. International Psychogeriatrics, 16, 295-315.

Brodaty, H. et al. (2001). Psychosis, depression and behavioral disturbances in Sydney nursing home residents: prevalence and predictors. International fournal of Geriatric Psychiatry, 16, 504-512.

Brodaty, H. et al. (2003). A randomized placebo-controlled trial of risperidone for the treatment of aggression, agitation, and psychosis of dementia. Fournal of Clinical Psychiatry, 64, 134 143.

Brylewski, J. and Duggan, L. (1999). Antipsychotic medication for challenging behaviour in people with intellectual disability: a systematic review of randomized trials. Fournal of Intellectual Disability Research, 43, 360-371.

Craig, D. et al. (2003). Factors influencing prescription of CNS medication in different elderly populations. Pharmacoepidemiology and Drug Safety, 12, 383-387.

De Deyn, P. et al. (1999). A randomized trial of risperidone, placebo, and haloperidole for behavioral symptoms of dementia. Neurology, 53, 946-955.

Folstein, M. F., Folstein, S. E. and McHugh, P. R. (1975). "Mini-mental state.” A practical method for grading the cognitive state of patients for the clinician. fournal of Psychiatric Research, 12, 189-198.

Gormley, N. and Howard, R. (1999). Should neuroleptics be used in the management of nursing home residents with dementia? International fournal of Geriatric Psychiatry, 14, 509-511.

Herrmann, N. and Lanctôt, K. (1997). From transmitter to treatment: the pharmacotherapy of behavioral disturbances in dementia. Canadian fournal of Psychiatry, 42, S51-S64.

Kallin, K., Gustafson, Y., Sandman, P. O. and Karlsson, S. (2005). Factors associated with falls among older, cognitively impaired people in geriatric care settings: a population-based study. American fournal of Geriatric Psychiatry, 13, 501-509.

Katz, I. (2004) Optimizing atypical antipsychotic treatment strategies in the elderly. Fournal of the American Geriatrics Society, 52, S272-S277.

Lindesay, J., Matthews, R. and Jagger, C. (2003). Factors associated with antipsychotic treatment drug use in residential care: changes between 1990 and 1997. International fournal of Geriatric Psychiatry, 18, 511-519.

Liperoti, R., Mor, V. and Lapane, K. (2003). The use of atypical antipsychotics in nursing homes. Fournal of Clinical Psychiatry, 64, 1106-1112.

Margallo-Lana, M. et al. (2001). Prevalence and pharmacological management of behavioral and psychological symptoms amongst dementia sufferers living in care environments. International fournal of Geriatric Psychiatry, 16, 39-44. 
Näsman, B., Bucht, G. and Eriksson, S. (1993). Behavioral symptoms in the institutionalized elderly: relationship to dementia. International fournal of Geriatric Psychiatry, 8, 843-849.

Pitkala, K. H., Laurila, J. V., Strandberg, T. E. and Tilvis, R. S. (2004). Behavioral symptoms and the administration of psychotropic drugs to aged patients with dementia in nursing homes and acute geriatric wards. International Psychogeriatrics, 16, 61-74.

Pollock, B. et al. (2002). Comparison of citalopram, perphenazine, and placebo for acute treatment of psychosis and behavioral disturbances in hospitalized, demented patients. American fournal of Psychiatry, 159, 460-465.

Robertson, J., Emerson, E., Gregory, N., Hatton, C., Kessissoglou, S. and Hallam, A. (2000). Receipt of psychotropic medication by people with intellectual disability in residential settings. Fournal of Intellectual Disability Research, 44, 666-676.

Rovner, B., Steele, C., Schmuely, Y. and Folstein, M. (1996). A randomized trial of dementia care in nursing homes. Fournal of the American Geriatrics Society, 44, 7-13.

Sandman, P. O., Adolfsson, R., Norberg, A., Nyström, L. and Winblad, B. (1988). Long-term care of the elderly. A descriptive study of 3600 institutionalized patients in the county of Västerbotten, Sweden. Comprehensive Gerontology, 2, 120-132.

Sloane, P. et al. (2004). Effect of person-centered showering and the towel bath on bathing-associated aggression, agitation, and discomfort in nursing home residents with dementia: a randomized, controlled trial. Fournal of the American Geriatrics Society, 52, 1795-1804.

Sørensen, L., Foldspang, A., Gulmann, N. and Munk-Jørgensen, P. (2001). Determinants for the use of psychotropics among nursing home residents. International fournal of Geriatric Psychiatry, 16, 147-154.

Statistical Package for Social Sciences Version II (for Mac OSX). SSPS, Chicago, IL, USA.

Thapa, P., Meador, K., Gideon, P., Fought, R. and Ray, W. (1994). Effects of antipsychotic withdrawal in elderly nursing home residents. Fournal of the American Geriatrics Society, 42, 280-286.

World Health Organization (2005). Anatomical Therapeutical Chemical (ATC) (Classification Index Including Defined Daily Doses (DDD) for Plain Substances. Oslo: World Health Organization Collaborating Center for Drug Statistics Methodology. 